\title{
Factores determinantes del uso de sorgo para alimentación de ganado bovino en el noroeste de México
}

Venancio Cuevas-Reyes a

Blanca Isabel Sánchez Toledano b*

Roselia Servín Juárez c

Juan Esteban Reyes Jiménez d

Alfredo Loaiza Meza d

Tomas Moreno Gallegos d

a Instituto Nacional de Investigaciones Forestales Agrícolas y Pecuarias (INIFAP). Campo Experimental Valle de México. Estado de México, México.

b INIFAP. Campo Experimental Zacatecas, Zacatecas, México.

c Colegio de Postgraduados Campus Córdoba, Córdoba, Veracruz, México.

d INIFAP. Campo Experimental Valle de Culiacán, Sinaloa, México.

*Autor de correspondencia: sugammx@ hotmail.com

Resumen:

El objetivo del estudio fue analizar los factores que determinan el uso de variedades de polinización libre de sorgo en el norte del estado de Sinaloa, con la finalidad de caracterizar el tipo de productores que utilizan este tipo de semillas. Se utilizó un modelo de elección discreta para identificar los factores que inciden en la adopción de sorgo de 199 ganaderos. Posteriormente, a través de pruebas no paramétricas se caracterizaron los adoptadores $(\mathrm{n}=$ 11) y no adoptadores $(n=188)$ de la tecnología. Los resultados muestran que $5.5 \%$ de los productores han adoptado variedades de sorgo. El número de años con asistencia técnica y 
la producción de leche resultaron significativas $(\mathrm{P}<0.05)$ para la adopción. En tanto, la caracterización de los ganaderos mostró que aquellos que cuentan con mayor cantidad de recursos: infraestructura, maquinaria, ganado, tierra, jornales y asistencia técnica, son los que adoptaron las variedades de sorgo. Se concluye que la adopción de semillas es baja y necesita de bienes públicos, como la extensión agrícola, para la difusión de sus beneficios que permita una mayor apropiación de los ganaderos en la región de estudio.

Palabras clave: Forraje, Polinización libre, Adopción, Innovación tecnológica, Doble propósito, Probit.

Recibido: 14/03/2019

Aceptado: 06/09/2019

\section{Introducción}

El sorgo es uno de los alimentos básicos para la población más pobre del mundo. Desde el punto de vista genético, este cultivo se adapta bien a zonas agroecológicas cálidas y secas en las que resulta difícil cultivar otros cereales. En muchas de esas zonas, tanto al grano como al forraje del sorgo se les concede un alto valor de uso $^{(1)}$.

México es el segundo productor de sorgo en el mundo con una participación del $10 \%$ de la producción mundial $^{(2)}$. En 2017, la superficie sembrada de sorgo en México fue de 1'456,330 ha, con una producción de 4'853,109 t. Dentro de los principales estados productores se encuentra Sinaloa, el cual ocupa el tercer lugar nacional en superficie sembrada con 109,382.59 ha ${ }^{(3)}$. En el año 2017, en Sinaloa se cultivó 1 millón 149 mil 320 ha, de las cuales el cultivo de hortalizas ocupó el $6.18 \%$ del total, los granos $67.52 \%$; oleaginosas $13.14 \%$; caña de azúcar $0.30 \%$; frutas $3.63 \%$; y, otros cultivos $9.24 \%$; el cultivo del sorgo representó el $14.10 \%$ de la superficie sembrada en el estado ${ }^{(3)}$.

Las variedades de sorgo de mayor uso en Sinaloa son materiales de "polinización libre": Costeño-201, Fortuna y Gavatero 203 del Instituto Nacional de Investigaciones Forestales, Agrícolas y Pecuarias (INIFAP) que han mostrado aceptación por los productores, y que al cosecharse se pueden utilizar para la siembra sin ser afectada la calidad genética del grano producido $^{(4)}$. A pesar de que el grano de sorgo es un importante alimento para animales, se le atribuye una "digestibilidad" baja respecto a otros cereales debido a la presencia de taninos condensados. Por lo general, los "taninos" se encuentran en granos de sorgo de color café, 
no así en sorgo de grano blanco, y escasamente en sorgo de grano rojo; en Sinaloa, la mayoría de las variedades de sorgo desarrolladas por el INIFAP son de grano blanco o crema ${ }^{(4)}$.

Estudios realizados sobre los factores que explican la baja adopción de variedades de semillas mejoradas identifican entre otros, la falta de adaptación de los materiales ofertados, la alta percepción del riesgo que conlleva su empleo al no conocer las ventajas de su uso en comparación con la usada por el productor, así como su deficiente distribución ${ }^{(5)}$. Algunas de las variedades generadas por el INIFAP y que actualmente son demandadas por los productores son: gavatero 203 , costeño 201 , sinaloense y en menor medida perla $101^{(6,7,8,9)}$. El sorgo gavatero 203 es el que ha tenido mayor aceptación por los productores; en el año 2016 se distribuyó semilla de esta variedad para la siembra de 70 mil ha de temporal en Sinaloa ${ }^{(10)}$.

El sorgo gavatero es de ciclo intermedio (61 días a la floración y 110 días a la cosecha), su grano es de color anaranjado rojizo, tiene un rendimiento de grano de $2,849 \mathrm{~kg} \mathrm{ha}^{-1}$ y 35,367 $\mathrm{kg} \mathrm{ha}^{-1}$ de forraje verde, además cuenta con $66.4 \%$ de digestibilidad y $7.3 \%$ de proteína ${ }^{(6)}$. El éxito logrado de esta variedad radica en su consistencia y buen comportamiento en condiciones de temporal $(350-600 \mathrm{~mm}$ ) y la aceptación del forraje por el ganado bovino. Aunado a que en condiciones adversas (sequía), la variedad se muestra vigorosa en etapas tempranas de desarrollo y su semilla se puede producir en cualquier época del año.

Para el caso de Sinaloa, la adopción de variedades de polinización libre de sorgo para ensilaje o forraje en utilización directa por el ganado bovino ha sido parcial y solo se ha avanzado en su uso en la zona centro y sur del estado, donde han sido realizadas las evaluaciones y demostraciones de siembra de este cultivo ${ }^{(6)}$. El presente estudio tiene como objetivo analizar los factores que determinan el uso de variedades de polinización libre de sorgo en el norte del estado de Sinaloa con la finalidad de caracterizar el tipo de productores que utilizan este tipo de semillas.

\section{Material y métodos}

\section{Localización de la zona de estudio}

Sinaloa se ubica dentro de la planicie costera noroccidental, la cual a su vez colinda directamente con la Sierra Madre Occidental. Geográficamente se localiza al noroeste de la República Mexicana, colindando al norte con los estados de Sonora y Chihuahua, al este con Durango, al sur con Nayarit y al oeste con el Océano Pacífico y Golfo de California (Figura 1), limitado por las coordenadas extremas $22^{\circ} 31^{\prime}$ y $26^{\circ} 56^{\prime} \mathrm{N}$ y ls $105^{\circ} 24^{\prime}$ y $109^{\circ} 27^{\prime} \mathrm{O}$ del meridiano de Greenwich ${ }^{(11)}$. 
Figura 1: Localización de la zona de estudio

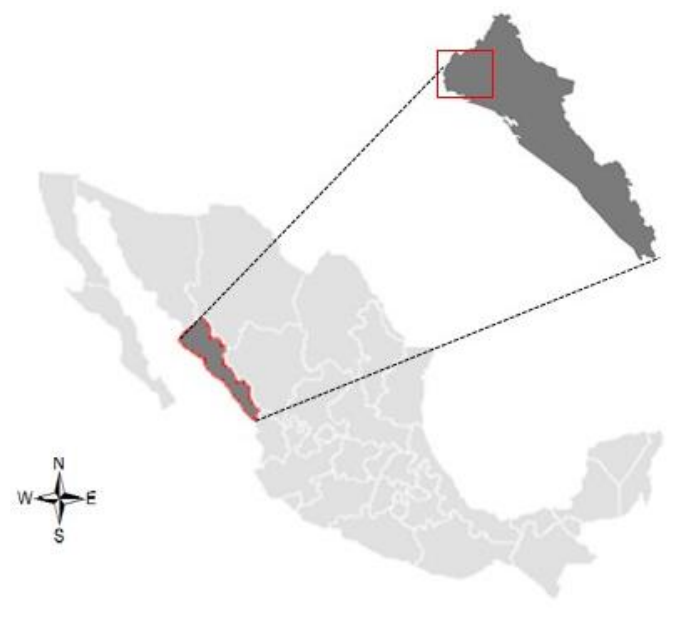

Colecta de datos

La información analizada proviene de una encuesta personal y cara a cara, realizada en 2015 , a una muestra de 214 productores del sistema bovinos de doble propósito (PSBDP) en el trópico seco de Sinaloa. Las encuestas se realizaron en el norte de Sinaloa, región que cuenta con tres municipios con condiciones climatológicas y geográficas favorables para la producción de sorgo: Ahome, El Fuerte y Guasave.

La selección de los productores se realizó por muestreo no probabilístico ${ }^{(12)}$. Para ello, se utilizaron los siguientes criterios de inclusión: a) ser propietario de ganado, b) no haber participado en programas de extensión agrícola previamente y, c) estar de acuerdo en responder la encuesta de diagnóstico inicial de su unidad de producción ganadera.

La encuesta consistió en recolectar datos de cada productor mediante un cuestionario con información estructurada en diez apartados, con preguntas de tipo cerrado y abierto, el cual se probó antes de su aplicación definitiva. Las variables incluidas en el cuestionario se dividieron en los siguientes apartados: 1) aspectos generales de la unidad productiva, 2) características sociales y económicas del productor, 3) tipo de propiedad de la superficie agrícola, 4) nivel de recursos disponibles (número de animales en posesión, tierras de uso agrícola, tierras de agostadero, fuentes de agua), 5) instalaciones disponibles (infraestructura, maquinaria y equipo), 6) aspectos sobre reproducción animal, 7) tipo de alimentación y suplementación del ganado, 8) aspectos relacionados con la sanidad pecuaria del hato, 9) aspectos relacionados con el manejo de ordeño y finalmente, 10) temas relacionadas con el mercado de productos pecuarios. En el análisis de la información se identificaron 15 encuestas con datos atípicos (por ejemplo, encuestas repetidas y datos fuera del promedio), por lo que solo fueron consideradas 199 encuestas con información confiable para el estudio. 
Esta encuesta fue parte de un proyecto que tenía como objetivo caracterizar los sistemas de producción pecuario en el norte de Sinaloa, los resultados de la caracterización, así como la descripción y cálculo del índice de infraestructura, maquinaria y equipo se describen en Cuevas-Reyes y Rosales-Nieto ${ }^{(13)}$.

\section{Modelo Probit}

El modelo Probit, es un modelo de elección discreta, el cual se caracteriza porque la variable dependiente toma dos únicos valores; 0 y $1^{(14)}$, que corresponden con cada una de las dos alternativas posibles (en este estudio: 1 adopta y 0 no adopta variedades de polinización libre de sorgo). El modelo utiliza una Función de Distribución Acumulativa (FDA) normal, de acuerdo a Gujarati ${ }^{(15)}$ la decisión de elegir una alternativa depende de un índice de conveniencia no observable $I_{i}$, determinado por una o diversas variables explicativas $\left(X_{i}\right)$, el índice $I_{i}$ puede ser expresado de la forma siguiente: $I_{i}=\beta_{0}+\beta_{k} X_{i}$.

Con el supuesto de normalidad, la probabilidad $\left(\mathrm{P}_{\mathrm{i}}\right)$ de que $I_{i}^{*}$ sea menor o igual que $I_{i}$ se calcula a partir de la FDA normal estándar como:

$$
\mathrm{P}_{\mathrm{i}}=\mathrm{P}[\mathrm{Y}=1 \mid \mathrm{X}]=\mathrm{P}\left(I_{i}^{*} \leq I_{i}\right)=\mathrm{P}\left(Z_{i} \leq \beta_{0}+\beta_{k} X_{i}\right)=\phi\left(\beta_{0}+\beta_{k} X_{i}\right)
$$

En la cual, $\mathrm{P}[\mathrm{Y}=1 \mid \mathrm{X}]$ significa la probabilidad de que un suceso ocurra dado el valor de $\mathrm{X}_{\mathrm{i}}$, $Z i$ es la variable normal estandarizada; es decir, $Z_{i} \sim \mathrm{N}\left(0, \sigma^{2}\right)$ y $\phi\left(\mathrm{X}_{\mathrm{i}} \beta\right)$ representa la distribución acumulativa (FDA) de una variable normal estándar ${ }^{(15)}$.

Para obtener información sobre $I$, así como $\beta_{0}$ y $\beta_{k}$ se toma la inversa de la ecuación (1):

$$
I_{i}=\phi^{-1}\left(I_{i}\right)=\phi^{-1}\left(P_{i}\right)=\beta_{0}+\beta_{k} X_{k i}
$$

En la cual:

$I_{i}=$ variable dependiente dicotómica que refleja la diferencia entre el uso y no uso de una tecnología (1 si la adopción de la variedad de sorgo tiene lugar, 0 en caso contrario).

$\phi^{-1}$ es la inversa de la FDA normal.

$\beta_{0}=$ es una constante.

$\beta_{k}, k=1,2 \ldots \mathrm{n}$ son los coeficientes de las variables independientes a estimar.

$X_{k i}=$ vector de variables exógenas que explican la adopción de las variedades de sorgo. 
Una vez estimado el modelo (1), si se toma la derivada parcial se obtiene ${ }^{(14)}$ :

$$
\frac{\partial \phi}{\partial X_{k}}=\phi\left(x_{k}^{i} \beta\right) \beta_{k}
$$

Así la ecuación (3) representa el efecto del cambio (probabilidad marginal) de una unidad de $X_{k i}$, sobre la probabilidad de que $\mathrm{I}=1$.

La estimación del modelo se realizó mediante el método de máxima verosimilitud, que proporciona estimadores consistentes y asintóticamente eficientes. Para contrastar la significancia individual de cada parámetro se utilizó el test de Wald, cuyo estadístico, z, sigue una distribución normal tipificada. En cuanto a la evaluación de la bondad global de los ajustes, se utilizó el $\mathrm{R}^{2}$ de McFadden, y el estadístico LR o razón de verosimilitud. Los resultados del modelo econométrico se obtuvieron en el paquete Data Analysis and Statistical Software (Stata) versión 12.

Una vez identificados los factores que determinan el uso de las variedades de sorgo, se procedió a caracterizar los productores que utilizan (o adoptan) las variedades de sorgo en comparación con los productores que decidieron no adoptarlas. Para analizar los grupos identificados se utilizaron estadísticas descriptivas, para las variables ordinales se aplicó la prueba de Mann-Whitney, toda vez que al realizar la prueba de normalidad a las variables cuantitativas éstas no cumplieron con la prueba de normalidad (Kolmogorov-Smirnov) de datos $(P<0.05)$, la información se procesó en el sistema de análisis estadístico SPSS $®$ versión 21 (IBM Corp).

\section{Resultados y discusión}

\section{Factores que determinan el uso de variedades de sorgo}

En el Cuadro 1 se muestran los resultados obtenidos del modelo probabilístico binario, el valor del Ji cuadrada se utilizó para el contraste de la significancia global del modelo; su hipótesis nula es que todos los coeficientes de la ecuación, excepto la constante, son nulos. El número de casos correctamente clasificados fue de $96.98 \%$, el estadístico LR $\mathrm{Ji}^{2}(9)$ fue de 40.04 y la probabilidad asociada fue menor a 0.05 , por lo que se rechaza la hipótesis nula, siendo de esta manera el modelo global estadísticamente significativo. El estadístico z aplicado a los coeficientes del modelo muestra que dos variables (asistencia técnica y la producción de leche) fueron significativas $(P<0.05)$ para explicar la probabilidad de adoptar variedades de sorgo en la región de estudio. 
Cuadro 1: Variables que influyen en la probabilidad de adoptar variedades de sorgo

\begin{tabular}{lllll}
\hline Variable & Coeficiente & $\mathbf{z}$ & $\boldsymbol{P > \mathbf { z }}$ & $\mathbf{d y} / \mathbf{d x}$ \\
\hline Índice de maquinaria y equipo, \% & 0.0057 & 0.47 & 0.639 & 0.00008 \\
Índice de infraestructura, \% & 0.0346 & 1.65 & 0.100 & 0.0004 \\
Asistencia técnica, años & 0.2149 & 2.47 & $0.013^{*}$ & 0.0030 \\
Edad, años & -0.0167 & -0.91 & 0.361 & -0.0002 \\
Escolaridad, años & -0.1470 & -1.19 & 0.234 & -0.0020 \\
Distancia al municipio, km & -0.0073 & -0.28 & 0.779 & -0.0001 \\
Número de vacas adultas & 0.0379 & 1.42 & 0.155 & 0.0005 \\
Superficie agrícola, ha & -0.0224 & -1.07 & 0.238 & -0.0003 \\
Producción de leche, L & -0.0306 & -2.46 & $0.014^{*}$ & 0.0004 \\
Constante & -1.6908 & -1.50 & 0.134 & \\
\hline
\end{tabular}

$\mathrm{dy} / \mathrm{dx}$ es el efecto marginal de la variable $\mathrm{x}$ sobre la variable dependiente $\mathrm{y}$; Nivel de significancia dy/dx: $P<0.05^{*}$. Numero de observaciones (n): 199. $\mathrm{LR} \mathrm{Ji}^{2}(9)=40.04$; Prob $>\mathrm{Ji}^{2}=0.0000$; Pseudo $\mathrm{R}^{2}=0.4706$,

Correctamente clasificados $=96.98 \%$.

Los efectos marginales de las variables significativas fueron pequeños, los PSBDP que cuentan con asistencia técnica tienen una probabilidad de $0.3 \%$ de adoptar nuevas variedades de sorgo, en tanto, la probabilidad de adoptar esta tecnología para los productores que desean incursionar en la producción de leche apenas es del $0.04 \%$.

Los resultados obtenidos en la variable de asistencia técnica en este estudio concuerdan con lo encontrado por otros autores ${ }^{(16-19)}$, quienes señalan la existencia de una relación positiva de la asistencia técnica con la adopción o uso de tecnología.

Un factor relevante en la adopción de nuevas variedades lo representa el precio de las semillas, el precio es un atributo extrínseco que afecta la decisión de compra del agricultor ${ }^{(20)}$. En el caso del sorgo Gavatero 203 el precio de compra durante el 2018 fue de $\$ 12.00$ por $\mathrm{kg}$, el cual tuvo el mismo precio que la variedad comercial sorgo "milon" (generación F2 o F3 de híbridos forrajeros Silo Miel, Cow Vittles u otros) que utilizan los productores. No obstante, al comparar ambos tipos de sorgo se observó que el sorgo Gavatero presenta mejor calidad, con $90 \%$ de semillas completas y viables, en comparación con $75 \%$ del "milon". Así mismo, cuenta con una mayor viabilidad de germinación (entre 85 y $90 \%$ ) en contraste con un 75 a $80 \%$ de otros sorgos ${ }^{(4)}$.

Una ventaja adicional de las variedades de polinización libre es su bajo precio respecto a los híbridos de sorgo. Al respecto, en la región de estudio existen semillas de híbridos de sorgo para ensilaje cuyo precio de venta al productor es de alrededor de $\$ 63.00$ pesos por kilo, este precio representa un $425 \%$ más alto respecto al precio $(\$ 12.00$ por $\mathrm{kg}$ ) de las variedades de polinización libre recomendadas en la zona de estudio. 
Estudios realizados sobre la adopción de variedades de maíz generados por el INIFAP, encontraron en el estado de Veracruz que las variables asociadas con la decisión del productor de utilizar semilla fueron: la cercanía del lugar de compra, el conocimiento de las semillas del INIFAP y en menor grado, la preferencia por sembrar semilla mejorada en sustitución de la variedad criolla ${ }^{(21)}$. Por otro lado, en la Península de Yucatán, a pesar de conocer las semillas mejoradas del INIFAP, el proceso de adopción se ve interrumpido por factores como la escasa disponibilidad de estas semillas en el mercado, o la falta de recurso económico por parte del productor para la adquisición de la semilla ${ }^{(22)}$.

Al igual que en Yucatán, uno de los factores que limita la adopción de sorgo en Sinaloa es la escasa disponibilidad y conocimiento de estos materiales por parte de los usuarios. Por lo que para mejorar la adopción se deben considerar los recursos disponibles y las decisiones de los productores $^{(23)}$, así como promover la reproducción de estas semillas a través de organizaciones de productores que cultiven u oferten más de un tipo de semilla y que reciban capacitación constante sobre tecnología de producción, beneficio, distribución, empaque y comercialización de variedades de polinización libre ${ }^{(24)}$.

La producción de sorgo para la alimentación del ganado en la región de estudio constituye una alternativa importante toda vez que al ser una variedad de polinización libre el mismo productor puede almacenar semilla para los siguientes ciclos. No obstante, para mantener su calidad genética se requiere de la producción controlada de este tipo de semillas. En la región de estudio la Fundación Produce Sinaloa es la única Institución que ha desarrollado un esquema de producción de algunas de las variedades de sorgo de polinización libre liberadas por el INIFAP, sin embargo, el mayor esfuerzo en la actualidad lo realizan productores individuales que por iniciativa y riesgo propio están incursionando en esta actividad para satisfacer la demanda de los PSBDP en todo el estado.

\section{Caracterización de adoptadores y no adoptadores de variedades de sorgo}

Los resultados obtenidos concuerdan con estudio previos que señalan que la adopción de

variedades de polinización libre por parte de los productores es baja ${ }^{(5,21,22)}$, al respecto, en el presente estudio solo $5.5 \%$ de los productores adoptó nuevas variedades de sorgo. A continuación, se caracteriza a los productores adoptantes y no adoptantes en sus aspectos sociales, productivos y tipos de productos obtenidos. 


\section{Aspectos sociales}

Las variables sociales analizadas entre el grupo de adoptadores y no adoptadores fueron iguales en ambos grupos $(P>0.05)$, por lo que desde el punto de vista social son familias con características similares (Cuadro 2). Estos resultados difieren a lo encontrado por otros autores ${ }^{(25)}$, quienes señalan que las características sociales son variables que diferencian a los usuarios de las innovaciones tecnológicas.

Cuadro 2: Variables sociales entre adoptadores y no adoptadores (mediana)

\begin{tabular}{llll}
\hline Variable & Adoptadores & No adoptadores & $\boldsymbol{P}^{*}$ \\
\hline Edad, años & 55.0 & 47.5 & 0.497 \\
Escolaridad, años & 3.0 & 3.0 & 0.389 \\
Dependientes mayores de 18 años, \# & 1.0 & 1.0 & 0.224 \\
\hline
\end{tabular}

*U de Mann-Whitney.

\section{Disponibilidad de recursos productivos}

En seis variables productivas se identificaron diferencias significativas $(P<0.05)$ entre los adoptadores y no adoptadores: índice de infraestructura, índice de maquinaria y equipo, tipo de jornal utilizado; eventual o asalariado, número de unidades animal, superficie total agrícola y número de años que ha contado con asistencia técnica. Es decir, los productores que adoptan las semillas de sorgo tienen más recursos productivos (superficie agrícola, número de unidades animal), mayor infraestructura y equipo, y además cuentan con mano de obra asalariada y asistencia técnica (Cuadro 3).

El mayor número de hectáreas lo tienen los adoptadores (mediana de 15 ha), en comparación con los no adoptadores (mediana de $8 \mathrm{ha}$ ); pareciera que la disponibilidad de tierra en conjunto con otros recursos productivos, hace menos adverso al riesgo al productor y con ello puede incursionar en la siembra de nuevas variedades.

Cuadro 3: Variables productivas entre adoptadores y no adoptadores (mediana)

\begin{tabular}{llll}
\hline Variable & Adoptadores & & No adoptadores \\
\hline Índice de infraestructura, \% & 36.36 & 18.18 & $\boldsymbol{P}^{*}$ \\
Índice de maquinaria y equipo, \% & 40.00 & 20.00 & 0.000 \\
Jornal (0= eventual; $1=$ contratado) & 1.00 & 0.00 & 0.006 \\
Total de vacas adultas, \# & 20.00 & 13.50 & 0.010 \\
Unidades animal, \# & 34.30 & 21.30 & 0.102 \\
Superficie agrícola, ha & 15.00 & 8.00 & 0.027 \\
Asistencia técnica, años & 4.00 & 0.00 & 0.029 \\
\hline
\end{tabular}

*U de Mann-Whitney. 
La adopción tecnológica se asocia positivamente con mayor tamaño de las parcelas y mayores ingresos ${ }^{(26)}$. Sin embargo, es normal que los agricultores suelan enfrentar cualquier innovación con incertidumbre y preconceptos sobre las afectaciones de las mismas ${ }^{(27)}$. Los resultados obtenidos respecto a las variables tamaño de la finca y contacto con servicios de asistencia técnica coinciden con diversos estudios realizados sobre el uso de variedades de arroz y trigo con pequeños productores ${ }^{(28,29)}$.

\section{Tipos de productos obtenidos: leche y carne}

Las variables explicativas producción de leche resultó significativa al 1\% ( $P=0.000)$ para los no adoptadores y al $5 \%(P=0.005)$ para la producción de becerros en el grupo de adoptadores. Este resultado indica que el grupo de adoptadores de nuevas variedades de sorgo están inclinados a la producción de becerros. Los adoptadores tuvieron una mediana de 0 y los no adoptadores una mediana de $30 \mathrm{~L}$ de leche. En contraste, la producción de becerros en los adoptadores tuvo una mediana de 2 y los no adoptadores una mediana de 0 . Parece ser entonces que el uso de nuevas variedades de sorgo tiene que ver con la posibilidad de contar con mayor cantidad de forraje para mejorar la producción de becerros para engorda.

El uso de variedades de polinización libre de sorgo en la zona de estudio presenta diversas ventajas respecto a otros materiales e híbridos, entre las cuales se puede citar; 1) mejor rendimiento en grano y forraje, 2) bajo precio respecto a los sorgos comerciales, 3) produce aún bajo condiciones de sequía, 4) el productor puede "cosechar" su semilla para el siguiente ciclo de siembra. Sin embargo, las variedades son poco conocidas por los productores. En este sentido, Kaliba et $a l^{(30)}$ mencionan que para lograr una mayor adopción de semillas de variedades de sorgo se necesita una vinculación entre la investigación, los servicios de extensión y los tomadores de decisión para promover tecnología agrícola apropiada de fácil acceso y actual a los productores que enfrentan restricciones de producción, mercado y acceso de información.

\section{Conclusiones e implicaciones}

En la región de estudio solo $5.5 \%$ de los productores decidieron utilizar variedades de polinización libre de sorgo como forraje para la alimentación del ganado. La asistencia técnica y la producción de leche fueron las variables que resultaron significativas para adoptar el uso de nuevas variedades de sorgo. Mediante la caracterización de los grupos analizados se pudo identificar que los productores que cuentan con asistencia técnica y mayor cantidad de recursos como tierra y ganado, tienen mayor disponibilidad a innovar con nuevas alternativas tecnológicas. Los resultados de la investigación permiten concluir que la adopción de variedades de polinización libre no es tan rápida debido a la poca disponibilidad de los nuevos materiales de sorgo, y a la falta de acciones de difusión y de transferencia de 
tecnología hacia los productores del sistema bovinos de doble propósito. Se requiere diseñar esquemas para la reproducción y difusión de semillas de sorgo de polinización libre, así como de otros bienes públicos, como la extensión agrícola, para que se pueda concretar la difusión y transferencia de estas innovaciones tecnológicas y lograr una mayor adopción por parte de los productores de ganado del norte de Sinaloa y en regiones con características y problemática similares.

\section{Agradecimientos}

Al INIFAP por el financiamiento otorgado de la presente investigación. El cual se enmarca en el proyecto "Evaluación del proceso de capacitación agropecuaria y uso de la tecnología promovida en los programas integrales de capacitación 2015-2018”. Con número SIGI:14462132918

\section{Literatura citada:}

1. FAO. Organización de las Naciones Unidas para la Alimentación y la Agricultura. La economía del sorgo y del mijo en el mundo: hechos, tendencias y perspectivas. Italia, 1997. http://www.fao.org/3/w1808s/w1808s00.htm\#Contents. Consultado 28 Feb, 2019.

2. FAOSTAT. Organización de las Naciones Unidas Para la Alimentación y la AgriculturaEstadísticas. 2019. http://faostat.fao.org. Consultado 5 Mar, 2019.

3. SIAP. Servicio de Información Agroalimentaria y Pesquera. Avances de Siembras y Cosechas por Estado y Año Agrícola. México. 2019. http:// www.gob.mx/siap. Consultado 6 Mar, 2019.

4. Palacios VO, Moreno GT, Loaiza MA, Reyes JJE, Medina ChS. Gavatero-203, Nueva variedad de sorgo forrajero para Sinaloa. INIFAP-CIRNO. Campo Experimental Valle de Culiacán. Folleto técnico No. 31. Culiacán, Sinaloa. 2009.

5. López PMA, García JC. Las industrias de la semilla de maíz en Brasil y México: desempeño anterior, problemas actuales y perspectivas para el futuro. Documento de Trabajo de Economía del CIMMYT 97-02. CIMYT. México. 1997.

6. Hernández ELA, Moreno GT, Loaiza MA, Reyes JJE. Gavatero-203, nueva variedad de sorgo forrajero para el estado de Sinaloa. Rev Mex Cienc Agric 2010;1(5):727-731.

7. Hernández ELA, Moreno GT, Loaiza MA, Reyes JJE. Sinaloense-202, nueva variedad de sorgo para el estado de Sinaloa. Rev Mex Cienc Agric 2010;1(5):733-737. 
8. Hernández ELA, Moreno GT, Loaiza MA, Reyes JJE. Perla-101: nueva variedad de sorgo para el estado de Sinaloa. Rev Mex Cienc Agric 2011;2(5):779-784.

9. Hernández ELA, Moreno GT, Loaiza MA, Reyes JJE. Costeño-201: nueva variedad de sorgo de temporal de doble propósito para Sinaloa. Rev Mex Cienc Agric 2011;2(5):785-790.

10. SENASICA. Servicio Nacional de Sanidad, Inocuidad y Calidad Agroalimentaria. Reporta el Cesavesin rendimientos de 10 toneladas por hectárea de maíz durante las primeras trillas en el estado. México. 2016. http://sinavef.senasica.gob.mx/ALERTAS/inicio/pages/single.php?noticia=1531. Consultado 5 Mar, 2019.

11. INEGI. Instituto Nacional de Estadística y Geografía. Anuario estadístico y geográfico $\begin{array}{llll}\text { de } & \text { Sinaloa } & 2017 . & \text { México }\end{array}$ https://www.datatur.sectur.gob.mx/ITxEF_Docs/SIN_ANUARIO_PDF.pdf.

Consultado 10 Ene, 2019.

12. Abascal FE, Grande El. Análisis de encuestas. España: Edit. ESIC; 2005.

13. Cuevas-Reyes V, Rosales-Nieto C. Caracterización del sistema bovino doble propósito en el noroeste de México: productores, recursos y problemática. Rev MVZ 2018;23(1):6448-6460.

14. Aldrich JH, Nelson FD. Linear Probability, Logit, and Probit Models. Newbury Park, California, USA: Sage Publications; 1984.

15. Gujarati DN, Porter DC. Econometría. México. McGraw Hill; 2010.

16. Galindo GG. Uso de Innovaciones en el grupo de ganaderos para la validación y transferencia de tecnología "Joachin", Veracruz, México. Terra 2001;(19):385-392.

17. McGinty MM, Swisher EM. Agroforestry adoption and maintenance: self-efficacy attitudes and socio-economic factors. Agrof Syst 2008;(73):99-108.

18. Solís D, Bravo-Ureta BE, Quiroga RE. Technical efficiency among peasant farmers participating in natural resource management programs in Central America. J Agric Econ 2009;60(1):202-219.

19. Cuevas RV, Baca MJ, Cervantes EF, Espinosa GJA, Aguilar AJ, Loaiza MA. Factores que determinan el uso de innovaciones tecnológicas en la ganadería de doble propósito en Sinaloa, México. Rev Mex Cienc Pecu 2013;4(1):31-46. 
20. Lockshin L, Jarvis W, D'Hauteville F, Perrouty J. Using simulations from discrete choice experiments to measure consumer sensitivity to brand, region, price, and awards in wine choice. Food Qual Prefer 2006;7(3-4):166-178.

21. Del Angel-Pérez A, Villagómez-Cortés J, Larqué-Saavedra B, Adame-García J, TapiaNaranjo C, Sangerman-Jarquin D, et al. Preferencias y percepciones asociadas con semilla mejorada de maíz según productores de Veracruz Central, México. Rev Mex Cienc Agric 2018;9(1):163-173.

22. Uzcanga PNG, Larqué SB, Del Ángel PAL, Rangel FMA, Cano GAJ. Preferencias de los agricultores por semillas mejoradas y nativas de maíz en la Península de Yucatán, México. Rev Mex Cienc Agric 2017;(5):1021-1033.

23. Sánchez-Toledano BI, Kallas Z, Gil JM. Importancia de los objetivos sociales, ambientales y económicos de los agricultores en la adopción de maíz mejorado en Chiapas, México. Rev Fac Cienc Agrar 2017;49(2):269-287.

24. Trejo HL, Gil MA, Sánchez HM, Carballo CA, López PA. Producción de semilla mejorada por organizaciones de agricultores caso "Productora de maíz teocintle". Rev Fitotec Mex 2004;27(1):93-100.

25. Sánchez-Toledano BI, Zegbe JA, Espinoza-Arellano JJ, Rumayor-Rodríguez AF. Adopción tecnológica de surcos-doble hilera con pileteo en cebada maltera. Trop Subtrop Agroecosyt 2017;20(1):25-33.

26. Chirwa, E. Adoption of fertilizer and hybrid seeds by smallholder maize farmers in Southern Malawi. Dev South Afr 2005;22(1):1-12.

27. Allub, L. Aversión al riesgo y adopción de innovaciones tecnológicas en pequeños productores rurales de zonas áridas: un enfoque causal. Est Soc 2001;(2):467-493.

28. Hagos H, Ndemo E, Yosuf, J. Factors affecting adoption of upland rice in Tselemti district, northern Ethiopia. Agric \& Food Secur 2018;7-59.

29. Chandio AA, Yuansheng J. Factors influencing the adoption of improved wheat varieties by rural households in Sindh, Pakistan. AIMS Agri Food 2018;3(3): 216-228.

30. Kaliba AR, Mazvimavi K, Gregory TL, Mgonja FM, Mgonja, M. Factors affecting adoption of improved sorghum varieties in Tanzania under information and capital constraints. Agric Econ 2018;6-18. 